\title{
Nuevas tecnologías, democracia y eficiencia de las instituciones políticas
}

\author{
Joan Oriol Prats \\ Universitat Autònoma de Barcelona \\ Instituto Internacional de Gobernabilidad \\ Universitat Oberta de Catalunya \\ joanoriol.prats@iigov.org
}

\section{Resumen}

En este artículo, se estudia el impacto que las tecnologías de la información y la comunicación (TIC) y el nivel de democracia que tienen sobre el desempeño de las instituciones políticas. En primer lugar, se estudia empíricamente el impacto que las TIC tienen sobre la eficacia de la acción de gobierno. A este respecto, se muestra estadísticamente para un conjunto de 98 países que la tecnología junto con el nivel de democracia son determinantes fundamentales de la calidad de la acción de gobierno, mientras que el nivel de apertura de la economía (como estimador de globalización) no resulta significativo. En segundo lugar, a partir del trabajo de Brynjolfsson y otros (2002), se identifican los determinantes organizativos de la asimilación eficiente de las TIC por las empresas y se aplican al caso de las principales instituciones políticas. En base a estos determinantes, se explica por qué los parlamentos, los partidos políticos y la Administración pública han asimilado las nuevas tecnologías cada uno de ellos de forma distinta, y en menor medida que muchas empresas. Finalmente, se expone la dirección de algunos cambios organizativos e institucionales necesarios para incorporar en mayor medida las nuevas tecnologías al proceso político y, así, mejorar su eficiencia.

Palabras clave: tecnologías de la información y la comunicación, democracia, eficacia del gobierno, instituciones políticas.

\section{Abstract. New technologies, democracy and the efficiency of political institutions}

This article studies the impact of information and communication technologies (ICTs) and democracy on political institutions performance. First, we study empirically the impact of technology on government efficiency. In this respect, we show statistically for a cross-section of 98 countries that ICTs and the democratic level significantly explain government performance, while economic openness (as a proxy for globalisation) does not. Secondly, taking Brynjolfsson et. al (2002) work we identify the organizational determinants of efficient ICTs assimilation by enterprises and we apply them to the main political institutions. Using these determinants, we explain why parliaments, political parties, and the public administration have assimilated technology in a distinct manner and path. Finally, we show the direction of some organizational and institutional changes

* El autor agradece el apoyo financiero prestado por el MCYT a través del proyecto SEC200309773-C02-01/CPSO 
needed in order to better incorporate technologies in the political process fostering then its efficiency.

Key words: information and communication technologies, democracy, government efficiency, political institutions.

\section{Sumario}

1. Introducción

2. Breve revisión de la literatura sobre tecnología, democracia e instituciones políticas

3. Eficacia gubernamental, tecnología y globalización
4. Los determinantes de la asimilación de tecnología por parte de las instituciones políticas

5. Conclusiones y futuras líneas de investigación

Bibliografía

\section{Introducción}

En el siguiente artículo, se pretende avanzar en el estudio de la relación entre tecnología e instituciones políticas. La tecnología ha sido un objeto de estudio poco frecuente en ciencia política y no ha sido hasta la aparición de las nuevas tecnologías de la información y el conocimiento que la tecnología ha cobrado una mayor relevancia en los estudios de ciencia política. Las potencialidades de las nuevas tecnologías para mejorar la transparencia, la participación e, incluso, la representatividad han generado un intenso debate a la luz de la interrelación entre tecnología e instituciones políticas.

El trabajo se compone de tres partes. En una primera parte se revisan brevemente los principales estudios sobre tecnología en las ciencias sociales, poniendo especial énfasis en aquéllos que han tratado de vincular las nuevas tecnologías con las instituciones democráticas. En segundo lugar se realiza un análisis empírico para analizar con más rigor el efecto de las nuevas tecnologías sobre la eficacia del gobierno. Las nuevas tecnologías contribuyen significativamente a mejorar la eficacia del gobierno, incluso controlando por los niveles de democracia, educación, renta y otros. Se plantean varios modelos y los resultados se muestran robustos ante distintas especificaciones. A raíz del análisis realizado, se argumenta que las nuevas tecnologías son en cierta medida «moralmente neutras" y pueden aprovecharse tanto por gobiernos democráticos como dictatoriales para mejorar su eficacia.

En tercer lugar se examinan cuáles son los determinantes organizativos de la asimilación eficiente de las nuevas tecnologías. Partiendo de la teoría de Brynjolfsson y otros (2000) y del supuesto de que la asimilación más eficiente de las nuevas tecnologías se ha producido hasta el momento por parte del sector privado, se analizan desde el institucionalismo político los determinantes organizativos que se han mostrado más eficientes en la asimilación de la tecnología. Estos determinantes se aplican al sector público para derivar las principales barreras a las que éste se enfrenta para utilizar más eficientemente la tecnología. Finalmente, se comentan un conjunto de hipótesis i líneas futu- 
ras de investigación sobre qué estructuras institucionales incentivan el uso de la tecnología.

\section{Breve revisión de la literatura sobre tecnología, democracia e instituciones políticas}

La relación de la tecnología con la democracia y las instituciones políticas de que se compone es compleja. En los últimos años, se han realizado varios esfuerzos desde las ciencias sociales para entender el papel de la tecnología en los procesos políticos, económicos y sociales. Los primeros estudios de reconocido impacto sobre el papel de la tecnología surgieron en el ámbito de la economía ya en la década de 1950 de la mano de Robert Solow, futuro premio Nobel de economía, quien remarcó y probó empíricamente la importancia de la tecnología para el crecimiento económico. Sin embargo, la tecnología de entonces hacía referencia a la «industrial», encaminada a sustituir la fuerza humana, y no a las denominadas «tecnologías de la información y la comunicación» (TIC), cuya finalidad es reemplazar las formas de comunicación existentes, el cálculo e incluso el pensamiento humano ${ }^{1}$. Las TIC son complejos sistemas nerviosos mucho más flexibles en cuanto a su diseño y a sus usos potenciales que la tecnología industrial tradicional. Es por este motivo que el análisis de la influencia de las nuevas tecnologías en el desarrollo ha de tomar en consideración que, en la actualidad, el impacto de la tecnología es de distinta índole y posee otras implicaciones organizativas y culturales más fuertes.

Como se ha dicho, desde la teoría macroeconómica la tecnología se vio como un motor del crecimiento económico. A su vez, también en la década de 1950, los estudios pioneros de Lipset (1959) relacionaron el crecimiento económico con el desarrollo de la democracia, lo que, unido al trabajo de Solow, ya deja entrever un potencial vínculo entre tecnología y democracia. Más recientemente, la teoría de la modernización considera que el crecimiento económico genera aspiraciones políticas en la población, que demanda mayores niveles de democracia y servicios públicos a medida que aumenta su bienestar económico (Welzel y Inglehart, 2001). De esta teoría se puede también establecer un vínculo indirecto positivo entre tecnología y democracia ${ }^{2}$.

Ya en los años ochenta, en la década de los ordenadores, se apreció uno de los factores más determinantes de las nuevas tecnologías. Con la frase "puedo ver los ordenadores en todas partes menos en las cifras de productividad ${ }^{3}$,

1. Véase, a este respecto, la extensa obra que Manuel Castells (1997) dedica al tránsito de la sociedad industrial a la sociedad informacional, donde se describe el cambio de tecnología acaecido y sus principales implicaciones para la estructura productiva, la cultura y la sociedad.

2. El vínculo a través del crecimiento económico es, no obstante, débil, en tanto otros autores, como Preworski y Limongi (1997), señalan que el desarrollo económico no es importante para la democratización. Sin embargo, más recientemente, otros autores como Boix y Stokes (2003) confirman la importancia del desarrollo económico para la democracia.

3. Esta cita fue recogida de un artículo del autor en el New York Times. Aquí se cita a partir de Sebastián Edwards (2001). 
Robert Solow expresaba la distancia que separaba la inversión en tecnología de sus rendimientos en términos de eficiencia. Esta idea ha sido compartida por economistas de reconocido prestigio, como Krugman (1994), desde una visión más histórica ${ }^{4}$. Una tecnología no se implanta completamente si se utiliza de forma aislada. Para aprovechar realmente los ordenadores y otras tecnologías, es necesario muchas veces reorganizar la estructura del trabajo dentro de la organización, así como la forma en que se relaciona dicha organización con el exterior. De esta manera, la asimilación de las nuevas tecnologías por las principales instituciones políticas es necesariamente un proceso lento que exige cambios institucionales y organizativos. Muchos de los cambios no se han emprendido por parte de las instituciones políticas más importantes del país, que tienen principios rectores distintos a los que rigen en las organizaciones empresariales de la llamada "nueva economía».

Conviene señalar, empero, que la aplicación de las nuevas tecnologías, tanto en las organizaciones privadas como públicas, exige un proceso reflexivo que requiere de cambios en los hábitos, las rutinas y los conocimientos compartidos de las organizaciones. Las organizaciones asimilan la tecnología bien porque consideran necesaria su introducción por razones de eficiencia o bien porque sus nuevas necesidades lo exigen. No obstante, a su vez, la tecnología también cambia las organizaciones y las instituciones, al requerir nuevos departamentos informáticos, nuevas formas de coordinación y comunicación entre empleados, nuevas formas de venta y de tratar con proveedores, y de otros muchos cambios de índole organizativa e institucional. La tecnología actúa, pues, como variable dependiente e independiente del desempeño de las organizaciones y las instituciones políticas (Fountain, 2001; Prats, 2002a). En este punto, se avanza con más profundidad en la segunda parte de este trabajo, donde se analizan los factores que han facilitado la asimilación eficiente de la tecnología en las empresas y se muestran las limitaciones inherentes a las instituciones políticas para la rápida y eficaz asimilación de la tecnología, así como las principales direcciones de los cambios que se necesitarían para superar dichas limitaciones.

Finalmente, conviene señalar otra última forma de estudio de las implicaciones de la tecnología para las instituciones políticas y que se ha producido a partir de la explosión del fenómeno concreto de Internet. Esta rama de investigación se refiere a la utilización de las nuevas tecnologías por parte de las instituciones públicas. De esta forma, existen estudios que van desde la utilización por parte de los poderes públicos de un procesador inteligente hasta la utilización de la televisión o Internet. Para estas aplicaciones, se ha acuñado el término «democracia electrónica» (Saldich, 1979), para el que existen varios sinó-

4. Algo similar pasó, no obstante, con otras innovaciones tecnológicas. Por ejemplo, aunque es razonable «afirmar que el adelanto tecnológico crucial que desencadenó la revolución industrial fue el invento de la hiladora de varios usos de Hargreave en 1764, [...] la industrialización general de Gran Bretaña no comenzó aproximadamente hasta 1810 y los salarios reales no empezaron a subir significativamente hasta la década de 1840. La energía eléctrica se introdujo en la década de 1880 y, sin embargo, el historiador Paul David ha afirmado que apenas influyó positivamente en la productividad hasta los años 20» (Krugman, 1994, p. 68). 


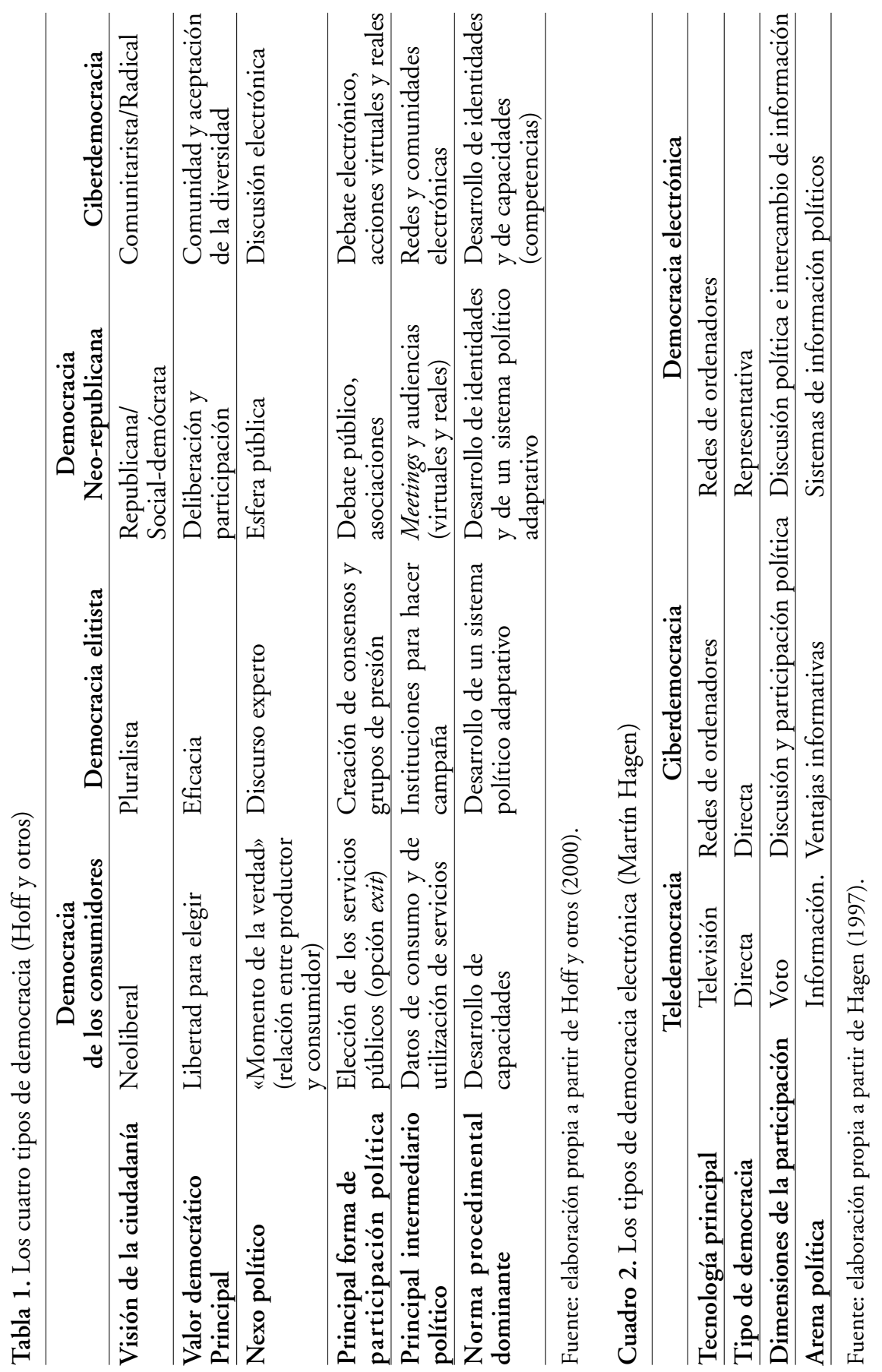


nimos, como el de «teledemocracia» (Etzioni, 1983; Corss, 1998), «democracia digital» (Cynthia y Leslie, 1998), "Cyberdemocracia» (Ogden, 1994; Poster, 1995), «democracia virtual» o «democracia de la sociedad de la información», que expresan el mismo concepto con ligeros matices según los autores.

A raíz de estos estudios, han surgido varias teorías sobre las implicaciones que la tecnología tiene para la democracia. A este respecto, destacan los trabajos realizados por Hoff y otros (2000) y por Martin Hagen (1997), quienes tratan de establecer tipologías de democracia en función de los artefactos tecnológicos aplicados (televisión, redes de computadores, etc.), los principales valores democráticos y el concepto de ciudadanía imperantes, la principal forma de participación política que conllevan (directa o representativa con más o menos deliberación), y algunos otros elementos (véanse tablas 1 y 2 para una visión sintética de los tipos de democracia planteados por estos autores) ${ }^{5}$. La utilidad de estos trabajos para nuestro estudio estriba en que dejan patente que las instituciones políticas pueden asimilar las nuevas tecnologías de múltiples formas. Sin embargo, el punto débil de estos estudios es que no están exentos de cierta "futurología», en tanto que la aplicación de las TIC en la práctica democrática no deja de ser todavía un misterio por resolver. Al mismo tiempo, no está claro que ciertas ideologías implementen en mayor medida unas tecnologías que otras, pues, como se argumenta más adelante, la tecnología es en gran parte moralmente neutra y puede servir por igual y de la misma forma a ideologías completamente distintas.

Para desenhebrar de forma más rigurosa los efectos de la tecnología sobre el desempeño de las instituciones públicas, a continuación se realiza un estudio empírico de la relación de la tecnología con la eficacia gubernamental. Mediante técnicas estadísticas, se estudia la contribución de la tecnología a la eficacia de la acción de gobierno en relación con otros factores importantes, como la democracia y la globalización.

\section{Eficacia gubernamental, tecnología y globalización}

¿El nivel tecnológico de un país influye sobre la calidad de la acción de gobierno? Los estudios realizados hasta la fecha no han abordado esta pregunta desde una aproximación empírica, debido en parte a la falta de datos sobre la eficacia del gobierno. Sin embargo, resulta importante conocer si la eficacia de las políticas públicas se ve afectada por el uso de las nuevas tecnologías en el país. A nivel teórico, es fácil caer en la cuenta que cuanto mayor es la dotación tecnológica de la que dispone un país, de más facilidades dispone su gobierno para emprender sus actividades con mayor eficacia y eficiencia. Los ejemplos son muchos. Los gobiernos pueden utilizar la tecnología para mejorar sus sistemas de gestión y supervisión financiera, impositiva, o de recursos humanos. Asimismo, también pueden implementar nuevos sistemas de comunicación

5. Puede obtenerse un resumen más extenso de los tipos de democracia electrónica planteados por estos autores en Prats y Del Álamo (2001). 
informática con los representados y administrados. Todas estas opciones derivan de la capacidad de acceder a la tecnología ${ }^{6}$.

La tecnología es, por tanto, un importante determinante de la capacidad del gobierno para llevar a cabo sus tareas. Es importante resaltar que, independientemente de cuáles sean los valores u objetivos que persiga el gobierno, nuestro supuesto es que el uso de la tecnología incrementa su eficacia. La tecnología es en este sentido moralmente neutra, puede utilizarse tanto para prohibir el acceso de miles de habitantes de China a gran parte de la información de Internet como para proveer información y conocimiento útil a muchos habitantes de forma más económica. Es, por tanto, perfectamente posible que un gobierno autoritario utilice las nuevas tecnologías de forma más eficaz que un gobierno democrático, pero sin embargo la democracia tiene otras virtudes para la eficacia gubernamental que van más allá del uso de la tecnología.

La democracia tiene otras virtudes que influyen por sí mismas en la eficacia del gobierno. Como han señalado varios autores, y muy especialmente Sen (2000) y Bardhan (2001), la democracia no sólo es un fin en sí misma, sino que también es un instrumento para el desarrollo. La democracia incrementa la eficacia de los gobiernos haciéndolos responsables ante la ciudadanía y proporcionando confianza en los mercados mediante una mayor transparencia. Con el tiempo, al tener que rendir cuentas cada cierto tiempo y estar sometidos a una mayor transparencia y escrutinio por parte de la sociedad civil, los gobiernos democráticos se vuelven más eficaces en el desempeño de sus labores. Esto no implica que la democracia no presente también puntos flacos que socaven el desarrollo. Por ejemplo, la pugna por la financiación de los partidos y las campañas electorales muchas veces conlleva que determinados grupos de presión capturen en su propio interés las decisiones públicas. Pero, en conjunto, los incentivos que la democracia provee a los gobernantes para actuar correctamente son mayores que los existentes en ausencia de democracia (Bardhan, 2001).

Otro elemento que comúnmente se ha relacionado con el desarrollo ha sido el nivel de apertura comercial de un país. Tal y como se ha demostrado, formar parte del mundo globalizado ayuda al desarrollo. Sin embargo, ¿son los países con un mayor nivel de apertura comercial significativamente más eficaces que los gobiernos más cerrados? A nivel teórico, los estudios más recientes demuestran que la apertura comercial fomenta el desarrollo vía su impacto positivo en la calidad de las instituciones y en su desempeño. Una mayor globalización de la actividad genera beneficios en términos de estabilidad de los derechos de propiedad y credibilidad de las instituciones — más allá de los beneficios en términos de productividad-, que ayudan al crecimiento eco-

6. Para un completo análisis del grado de asimilación que un gobierno puede realizar de las dotaciones tecnológicas existentes, véanse los estudios de E-preparación elaborados por la Universidad de Harvard (http://cyber.law.harvard.edu/readinessguide/), la consultora Price Waterhause (http://www.ereadiness.pwcglobal.com/) y otras varias. Un extenso manual metodológico de cómo realizar una e-readiness puede encontrarse en $2002 \mathrm{~b}$. 
nómico. Así, los beneficios derivados de la apertura dependen sobre todo del nivel de renta del país. Los países más ricos tienen más probabilidades de aprovechar más la apertura comercial en términos de crecimiento que los pobres (Edwards, 2000; Rodrik y otros, 2002).

Desde otro punto de vista, autores como Dani Rodrik (2001) señalan la supremacía de las instituciones sobre la globalización a la hora de determinar el grado de disciplina y calidad de las políticas públicas. Como señala el mismo Rodrik, "las instituciones financieras internacionales señalan cada vez más la "disciplina de mercado" como el ingrediente crítico en el diseño de las políticas y como un importante argumento a favor de la libre circulación de capitales. Esta perspectiva subestima los beneficios de la democracia y sobreestima la bondad de la "disciplina de mercado" [...] La democracia es el mejor garante de la buena gobernanza, tanto en la esfera económica como política. Las libertades civiles y políticas, y los procedimientos participativos son la mejor manera de asegurar estándares laborales adecuados, estabilidad económica o sostenibilidad medioambiental. [...] Dadas estas circunstancias, la reafirmación de la primacía de la disciplina democrática sobre la disciplina de mercado debe ser fuerte, clara y frecuente» (Rodrik, 2002, p. 1).

Para contrastar esta hipótesis y observar el papel de la tecnología sobre la eficacia del gobierno, se realiza una regresión lineal múltiple donde la variable dependiente es la eficacia del gobierno y las variables independientes o explicativas son la tecnología, el nivel de democracia, el nivel de apertura comercial, la tasa de alfabetización y el PIB por habitante del país. Estas dos últimas variables se utilizan para controlar por el nivel de capital humano y la renta. La muestra alberga un conjunto de más de 98 países de todo el mundo y los datos han sido obtenidos de la Shared Global Database ${ }^{7}$ (véase el anexo 1 para una relación de fuentes).

Como estimador de la eficacia del gobierno, se ha utilizado el índice elaborado por Kaufmann y otros (2002). Estos autores obtienen indicadores para cada una de las dimensiones de la gobernanza que identifican mediante un análisis cluster en base a indicadores sobre desempeño institucional obtenidos de más de 18 fuentes distintas y utilizando un sistema de estimación de componentes no observados ${ }^{8}$. El índice de Eficacia Gubernamental combina las percepciones sobre la calidad de la provisión de los servicios públicos y de la

7. Esta base de datos puede descargarse gratuitamente de la página web de la profesora de Harvard Pipa Norris. Disponible en http://ksghome.harvard.edu/ - pnorris/Data/Data.htm.

8. Kaufmann, Kraay y Pablo Zoido-Lobatón (2002, p. 4) entienden por gobernanza «las tradiciones y las instituciones mediante las que se ejerce la autoridad en un país». Estos autores organizan el gran conjunto de variables que integran la misma en tres grandes clusters, a saber: (1) el proceso mediante el cual el gobierno es escogido, reemplazado o reelegido, y supervisado; (2) la capacidad del gobierno para formular e implementar políticas adecuadas, i (3) el respeto a las instituciones que rigen las interacciones económicas y sociales entre los ciudadanos y el Estado. El indicador de eficacia del gobierno utilizado en el análisis pertenece al segundo cluster, mientras que el de estabilidad política y el de Estado de derecho pertenecen al primero y al tercero respectivamente. 
burocracia, la competencia e independencia respecto a las presiones políticas de los funcionarios públicos y la credibilidad de los compromisos públicos. También para medir la eficacia del gobierno se utiliza el índice de corrupción elaborado por estos mismos autores en base a percepciones.

Como se observa en la tabla 2 siguiente, los determinantes de la eficacia del gobierno más significativos son el índice de nuevas tecnologías, el nivel de democracia (tal y como pronosticaba la hipótesis de Rodrik) y el PIB per cápita del país. La apertura del país al exterior no parece explicar de manera significativa la calidad institucional, lo que pone en duda los pretendidos efectos beneficiosos que la globalización tiene sobre la eficiencia de las instituciones 9 .

Tabla 2. Eficacia del gobierno, nuevas tecnologías y democracia.

Resultados de la regresión lineal múltiple

\section{Variables dependientes}

\begin{tabular}{lcccc} 
& $\begin{array}{c}\text { Modelo I } \\
\text { Eficacia } \\
\text { del gobierno }\end{array}$ & $\begin{array}{c}\text { Modelo II } \\
\text { Eficacia } \\
\text { del gobierno }\end{array}$ & $\begin{array}{c}\text { Modelo III } \\
\text { Corrupción }\end{array}$ & $\begin{array}{c}\text { Modelo IV } \\
\text { Inestabilidad } \\
\text { política }\end{array}$ \\
\hline Variables independientes & 0,112 & 0,433 & $-0,154$ & $-0,202$ \\
Constante & $(0,359)$ & $(1,028)$ & $(-0,533)$ & $(-0,527)$ \\
\hline Índice de nuevas & $0,095^{*}$ & $0,091^{* *}$ & $0,145^{*}$ & 0,006 \\
tecnologías (1) & $(2,394)$ & $(2,110)$ & $(3,926)$ & $(0,565)$ \\
\hline Índice de autocracia/ & $-0,134^{*}$ & $-0,144^{*}$ & $-0,138^{*}$ & $-0,151^{*}$ \\
democracia/(2) & $(-3,468)$ & $(-2,529)$ & $(-3,866)$ & $(-3,194)$ \\
\hline PIB per cápita & $0,002765^{*}$ & $0,0026^{* *}$ & $0,0145^{*}$ & $0,0062^{* *}$ \\
& $(2,790)$ & $(1,421)$ & $(1,635)$ & $(1,794)$ \\
\hline Exportaciones de bienes & 0,00 & & $-0,001$ & 0,000 \\
y servicios (\% PIB) & $(0,054)$ & & $(-0,018)$ & $(-0,348)$ \\
\hline Sector agrícola (\% PIB) & & $-0,0087$ & & \\
\hline Tasa de alfabetización & 0,120 & $-0,0019$ & 0,0037 & $-0,002$ \\
& $(1,791)$ & $(-0,569)$ & $(1,376)$ & $(-0,019)$ \\
\hline $\mathbf{N}$ (observaciones) & 98 & 80 & 98 & 98 \\
\hline $\mathbf{R}$ 2 ajustado & 0,715 & 0,596 & 0,781 & 0,564 \\
\hline
\end{tabular}

(1) El índice de nuevas tecnologías engloba el tanto por ciento de conectados a Internet, el tanto por ciento de PC y el tanto por ciento de hosts. (2) El índice de democracia tiene una escala inversa (10 autocracia, 0 democracia) que ha de tenerse en cuenta para leer los resultados. Los $\operatorname{signos}^{* *} \mathrm{y}^{*}$ corresponden a $\alpha 5$ y $1 \%$ respectivamente. Los valores entre paréntesis representan a los estadísticos $t$ de la región lineal múltiple.

9. Esto puede deberse en parte a los efectos que la apertura comercial tiene directamente sobre el crecimiento del PIB per cápita o a la forma de medir la globalización con el porcentaje de exportaciones respecto al PIB. Otras especificaciones quizás encuentren resultados distintos, ésta es una línea que se deja abierta a futuras investigaciones. 
Para mejorar la robustez de la especificación estadística empleada, se elaboran cuatro modelos. El primero de ellos utiliza como es el modelo original y sus resultados confirman la hipótesis de que la eficacia del gobierno depende más del nivel de democracia y de la introducción de tecnología que del nivel de globalización de la economía. Como se observa, estos resultados se mantienen en el modelo II, donde, en vez de utilizar como variable independiente el porcentaje del exportaciones respecto al PIB, se utiliza el porcentaje del sector agrícola, que sigue sin explicar significativamente el nivel de desempeño institucional. En el modelo III se cambia la variable dependiente, que pasa a ser la corrupción. Cuando realizamos este ejercicio, los resultados obtenidos se mantienen, lo que resulta consistente, ya que la corrupción es una variable clave de la eficacia gubernamental (sobre todo a nivel de percepciones, que es como se miden estas variables). Sin embargo, en el modelo IV la variable dependiente es la estabilidad política. En este modelo, la tecnología ya no resulta una variable relevante, mientras que la democracia y la renta del país sí se muestran como variables fuertemente relevantes. Esto último resulta consistente con la teoría en tanto, si bien la eficacia de las políticas y la corrupción sí dependen de la eficiencia inducida tecnológicamente, la inestabilidad política depende de otros factores diferentes.

$\mathrm{El}$ análisis de regresión realizado se somete a varios tests para examinar su correcta especificación. En primer lugar, se ha confirmado que la regresión no varía cuando se utiliza el estimador de White para controlar problemas de heterocedasticidad. En segundo lugar, para detectar posibles problemas de multicolinealidad, se analizan los incrementos del factor de la varianza (FIV). Como los FIV son inferiores a 5 en la mayoría de los modelos, la multicolinealidad no es excesiva. Los principales problemas de multicolinealidad se dan principalmente entre las nuevas tecnologías y el PIB per cápita, lo que exigiría de futuros análisis que, por ejemplo, introdujeran variables instrumentales para evitar la correlación entre el producto interior bruto y las nuevas tecnologías. En futuros estudios convendría utilizar otros indicadores y controlar por medio de otros factores relevantes para la eficacia del gobierno señalados por la literatura especializada (origen legal, exportaciones de petróleo y materias primas, etc.).

\section{Los determinantes de la asimilación de tecnología por parte de las instituciones políticas}

$\mathrm{Si}$, tal y como se ha mostrado, la tecnología mejora la eficacia de la acción del gobierno, ¿qué organizaciones son más proclives a asimilar las nuevas tecnologías de forma más eficiente? A continuación, se intenta indagar en esta cuestión a partir de los estudios que muestran los diseños organizativos que han asimilado de forma más productiva las nuevas tecnologías en la empresa privada. Estos estudios pioneros tratan de encontrar los factores explicativos de por qué unas empresas han asimilado de forma más eficiente las nuevas tecnologías que otras. Nuestro ejercicio estriba en, a partir de los factores detectados por la literatura en organización empresarial y nuevas tecnologías, explicar por qué 
unas instituciones políticas han asimilado las nuevas tecnologías en mayor medida que otras. Así, se explica por qué los partidos políticos y el Parlamento van con retraso en asimilación tecnológica con respecto a la Administración pública y por qué el sector público en general va con retraso respecto al privado. Esta explicación es fundamentalmente teórica. Dejaremos el ejercicio de su validación empírica para estudios subsiguientes.

Como se ha dicho al principio, los economistas y politólogos se han mostrado durante mucho tiempo escépticos a las repercusiones de la tecnología sobre sus respectivas áreas de trabajo. Este escepticismo queda reflejado en la citada célebre frase del Premio Nobel Robert Solow, quien, en 1987, afirmara «los ordenadores pueden verse en todas partes, menos en las cifras de productivi$\mathrm{dad} »^{10}$. Esta afirmación respondía a la inquietud que despertaba en muchos economistas el observar que la contribución de la inversión en ordenadores a la productividad hasta mediados de la década de 1990 había sido muy pequeña (Jorgensson y Stiroh, 1995).

Sin embargo, a finales del siglo pasado nuevos estudios demostraron que la mitad del aumento en la productividad experimentado por los Estados Unidos durante la segunda mitad de los noventa se debía a la inversión en nuevas tecnologías (Oliner y Sichel, 2000; Nordhaus, 2001). Esto indica que existe una brecha entre el momento en que se realizan las primeras inversiones en equipos y conocimientos informáticos y aquel otro momento en que se recogen los beneficios. Es probable que entre estos dos momentos exista también algún tipo de cambio organizativo o de índole institucional que explique también el éxito en la asimilación de las nuevas tecnologías por el proceso productivo. Una distinción importante que realizan los estudios sobre el impacto agregado de la tecnología en la economía es entre los sectores que «usan ordenadores» y aquellos que «fabrican» ordenadores. Una conclusión importante de los estudios sobre el impacto económico de la tecnología es que el impacto sobre la productividad de los sectores que fabrican es significativamente mayor que aquéllos que usan ordenadores (Edwards, 2001).

Más allá de tener presente los resultados estadísticos obtenidos a nivel agregado, resulta interesante examinar los principales estudios que a nivel micro se han emprendido sobre sectores productivos y empresas individuales para conocer los mecanismos organizativos particulares mediante los que la inversión en tecnología afecta a la productividad y al crecimiento. Siguiendo los principales investigadores del MIT al respecto, Brynjolfsson y Hitt (2000) y Greenan y otros (2001), se puede decir que el impacto de las tecnologías sobre la productividad es indirecto y está relacionado principalmente con cambios en otros aspectos del proceso productivo.

Como señalan los propios autores «la inversión en tecnologías de la información complementa los cambios en otros aspectos de la organización [...] Para tener éxito, es habitual que las empresas tengan que instalar ordenadores como parte de un "sistema" o "conjunto" de cambios organizativos que se 
refuerzan mutuamente [...] La realización de una inversión en ordenadores sin un cambio organizativo o con la sola introducción parcial de algunos cambios organizativos, puede acarrear pérdidas de productividad significativas» (Brynjolfsson y Hitt, 2000, p. 25).

En base a estudios empíricos en diversos sectores productivos, estos mismos autores señalan que la inversión en tecnologías de la información y la comunicación tienen mayor impacto en (a) entornos de trabajo descentralizados o existe una menor integración vertical; (b) en empresas de nueva creación, y (c) allí donde las relaciones laborales presentan una flexibilidad mayor.

Examinando los factores señalados que conducen a un aumento del impacto de la asimilación de nuevas tecnologías en la productividad, observamos que su aplicación a las principales instituciones y organizaciones políticas explica bastante bien por qué éstas han asimilado con retraso y sin demasiada eficacia las nuevas tecnologías y hacia dónde resultaría posible avanzar para superar los obstáculos que frenan el aprovechamiento de estas tecnologías por parte de los poderes públicos y las organizaciones políticas.

En primer lugar, los entornos de trabajo en los partidos políticos y en los parlamentos no se caracterizan por su elevado nivel de descentralización en sus actividades. En cambio, en la Administración pública, donde los entornos de trabajo descentralizados y la especialización del trabajo es mayor, los beneficios de la aplicación de las nuevas tecnologías se han dejado notar más, como claramente sucede en la gestión y la recaudación de impuestos.

La integración vertical de los partidos políticos y de los parlamentos es fuerte, puesto que ha de existir una única institución responsable de la acción legislativa o de la elaboración de un programa electoral. Otra vez, en la Administración pública es más factible que sí exista desintegración vertical, en tanto la Administración es más libre para subcontratar servicios que puede proveer el mercado.

La nueva creación de partidos políticos o de instituciones legislativas es prácticamente nula. Existen barreras a la entrada de tipo institucional (como el sistema electoral), económicas (financiación de los partidos) y derivadas del comportamiento político (vínculos actitudinales de los ciudadanos hacia los partidos) a la creación de nuevos partidos que reducen los incentivos que las nuevas tecnologías proveen a la creación de nuevas instituciones. Otra vez más, en la Administración pública se crean nuevas organizaciones de forma constante a medida que las nuevas necesidades ciudadanas o la propia eficiencia de la Administración lo requieren.

Finalmente, en contraste, la flexibilidad de las relaciones laborales en el seno de los partidos y las cámaras legislativas es quizás mayor que en la Administración pública. No obstante, los rígidos lazos informales que muchas veces prevalecen en los partidos y también en los legislativos, provoca que, en relación con el mercado, las relación laborales en las instituciones públicas sean bastante rígidas. A su vez, la rigidez laboral de la Administración pública podría explicar en gran parte por qué ésta ha asimilado con retraso las nuevas tecnologías en relación con el mercado. 
De esta forma, para mejorar la eficacia de la introducción de las nuevas tecnologías en las principales instituciones políticas, serían necesarios cambios organizativos que tendieran a descentralizar las actividades, a reducir las barreras a la entrada a la formulación de políticas y la representación ciudadana, y a flexibilizar las relaciones laborales. Para ello, resultaría necesario avanzar en las siguientes líneas, muchas de las cuales ya se están produciendo de manera espontánea.

La descentralización de las actividades en los parlamentos pasa, por ejemplo, por un papel más importante de las comisiones parlamentarias, que, si tuvieran un mayor peso en el proceso legislativo, tendrían más incentivos y presiones a utilizar las nuevas tecnologías para abrirse a la participación ciudadana, recoger opiniones de expertos, colgar sus deliberaciones y propuestas en la red, y disponer de sistemas de información y de conocimiento propios sobre temas específicos. En Estados Unidos, donde las comisiones parlamentarias son más importantes, el nivel de informatización y digitalización del parlamento es mayor ${ }^{11}$.

En cuanto a los partidos, la descentralización presenta mayores dificultades en tanto puede ir en contra de la unidad del propio partido. La descentralización de los partidos conlleva la creación de unidades internas de pensamiento independiente (think tanks) que pueden ir en contra de la imagen pública del partido. Resulta curioso que en los partidos con mayor indisciplina, como en los Estados Unidos, la utilización de las nuevas tecnologías haya sido mayor. Así, por ejemplo, y seguramente debido también a otros factores, en marzo del 2000 el Partido Demócrata de Arizona utilizó el voto electrónico en sus elecciones primarias, con lo que se convirtió en el primer sufragio legalmente reconocido donde podía votarse por Internet.

A nivel más general, es probable que la democratización interna de los partidos políticos pueda actuar como incentivo al fortalecimiento de los requisitos de transparencia y para un mayor debate público sobre modelos alternativos de política. Las nuevas tecnologías proveen a la vez de formas de organizar la información y ponerla a disposición de un público que gana capacidad de seguimiento, a la vez que adquiere nuevas formas de participación y comunicación a menor costo. La reducción del coste de participación y una mayor información se han de ver acompañadas de una capacidad de influencia política, que aumenta en entornos descentralizados que amplían los nodos de decisión.

Las barreras a la entrada son otro de los elementos que diferencian a las organizaciones que mejor han aprovechado las nuevas tecnologías de aquéllas que no lo han logrado. Las barreras a la entrada al proceso político son fundamentalmente de dos tipos: barreras a la formación de organizaciones políticas y barreras a la participación en la formulación de políticas. Como se ha dicho,

11. Para un ránquing del nivel de informatización de los parlamentos o para realizar comparaciones entre unos y otros, véase la página web del Cyberspace Policy Research Group (www.cprg.org). 
las barreras a la formación de partidos políticos vienen en gran parte explicadas por dimensiones: socioeconómicas y culturales (cleavages) e institucionales.

Conviene tener presente que las principales entidades políticas de «nueva creación» sí se han aprovechado en mayor medida de las nuevas tecnologías que los «viejos» partidos. Aunque el carácter de oligopolio del sistema de partidos ha impedido nuevas entradas, la actuación política ha cobrado nuevas formas. Las organizaciones no gubernamentales (ONG) han canalizado nuevas preferencias de las personas y han actuado con vocación política fuera del espectro del sistema tradicional de partidos. Son precisamente estas organizaciones las que con mayor eficacia han utilizado las nuevas tecnologías para desarrollar sus actividades. El abaratamiento de las necesidades de coordinación y gestión que supone la utilización de las nuevas tecnologías facilita la acción colectiva de organizaciones no gubernamentales atomizadas. De esta manera, allí donde las barreras institucionales lo permiten, al igual que en el mundo de la empresa, las nuevas entidades políticas tratan de explotar en mayor medida y con más eficiencia las ventajas ofrecidas por las nuevas tecnologías.

Las barreras a la formulación de las políticas públicas se deben a la apropiación del proceso político por los partidos y los principales grupos de interés y de presión, a la falta de mecanismos institucionales de participación ciudadana directa y a la propia ausencia de motivación por parte de muchos ciudadanos para los que el costo económico o en términos de ocio de la participación política es muy elevado. Poco a poco, nuevas experiencias de participación ciudadana se están emprendiendo en todo el mundo, sobre todo a nivel local. Las iniciativas de participación que primero surgieron en Brasil pueden suponer un buen incentivo a la mayor utilización de las nuevas tecnologías por parte de los poderes públicos, para facilitar la participación y proveer de información a la ciudadanía sobre los aspectos sujetos a deliberación. Estas nuevas formas de participación ciudadana, cuando superen el reto de la conexión universal, pueden resultar una fórmula eficaz para utilizar las nuevas tecnologías en el proceso político.

Otra cuestión que se plantea a raíz del papel que las nuevas tecnologías tienen para la canalización de las preferencias ciudadanas es si aquéllas, tal y como ha sucedido en el mercado de bienes (Brynjolfsson y otros, 2003), también permiten una diversificación del producto y el consiguiente aumento del bienestar de los consumidores. Existen especificidades del proceso político que hacen pensar que las nuevas tecnologías en el sistema político no puedan incorporar o satisfacer más las preferencias ciudadanas de lo que ya lo están actualmente. Sin embargo, sí existen cambios organizativos complementarios que diversifican la participación y proveen de diferentes tipos de información política relevante para que las personas puedan participar en aquello que es de su mayor interés, con lo que se aumentan la cultura y el activismo político.

Finalmente, también la flexibilidad de las relaciones laborales se ha probado como un factor determinante del grado de eficacia con el que se uti- 
lizan las nuevas tecnologías. La renovación constante del equipo y los conocimientos informáticos no sólo necesitan de un entorno descentralizado y abierto donde la especialización se combine con la innovación, sino también de un personal preparado y con una elevada movilidad. La movilidad laboral y la flexibilidad en el mercado de trabajo explican parte del retraso que, por ejemplo, ha sufrido la Administración pública en relación con el sector privado en cuanto a la adopción de nuevas tecnologías se refiere. Un mercado de laboral flexible estimula la renovación constante de los conocimientos del trabajador, así como la contratación de trabajadores con nuevas aptitudes.

Así pues, se han tratado de explicar los problemas que han tenido las principales instituciones políticas a la hora de incorporar la tecnología en sus actividades y el poco éxito logrado hasta la fecha en relación con el mercado. En este sentido, se ha señalado como las barreras a la entrada al «mercado político», la falta de descentralización y apertura de las actividades políticas y la rigidez de las relaciones laborales han actuado como freno a la asimilación de las nuevas tecnologías. A su vez, recurriendo a estos factores, se ha explicado por qué la Administración pública ha tenido mayores problemas que el sector privado para incorporar la tecnologías, pero menos que las principales instituciones políticas: partidos políticos y cámaras legislativas.

\section{Conclusiones y futuras líneas de investigación}

En este breve trabajo se ha mostrado la influencia que la tecnología tiene en la acción de gobierno a dos niveles: un nivel macro y un nivel micro. A nivel más general se ha probado, mediante un análisis de regresión múltiple, que la eficacia del gobierno se beneficia de las dotaciones tecnológicas del país al igual que de la democracia, mientras que el grado de apertura comercial (su nivel de inserción en la sociedad globalizada) no era un parámetro significativo para explicar la eficacia de un gobierno, medida tanto por la calidad de los servicios públicos como por la ausencia de corrupción.

A la luz de los beneficios que la tecnología presenta en términos de eficacia, nos preguntamos cuáles son los factores que explican que unas organizaciones asimilen las nuevas tecnologías de forma más eficiente que otras. A este respecto, se observa cómo las instituciones políticas cuentan con pocos de estos atributos, puesto que no están descentralizadas y sí integradas verticalmente, no tienen relaciones laborales flexibles y no son de nueva creación. Para superar estos defectos, las instituciones políticas deberían abrirse más a la sociedad democratizando los partidos, incluyendo mecanismos participativos y deliberativos en la formulación de políticas y descentralizando las instancias legislativas, a fin de generar la necesidad de sistemas de información política e incentivar su apertura mediante el uso de las nuevas tecnologías. 


\section{Anexo I. Relación de variables y fuentes}

\begin{tabular}{ll}
\hline Indicadores & Fuentes \\
\hline Eficacia del Gobierno 1997/8 & $\begin{array}{l}\text { Kaufmann y otros: World Governance } \\
\text { Indicators. }\end{array}$ \\
\hline Corrupción 1997/8 & $\begin{array}{l}\text { Kaufmann y otros: World Governance } \\
\text { Indicators. }\end{array}$ \\
\hline Estabilidad Política 1997/8 & $\begin{array}{l}\text { Kaufmann y otros: World Governance } \\
\text { Indicators. }\end{array}$ \\
\hline $\begin{array}{l}\text { Indicador de autocracia/democracia } \\
1990-5\end{array}$ & Freedom House. \\
\hline Tasa de alfabetización 1997 & $\begin{array}{l}\text { World Development Indicators, Banco } \\
\text { Mundial. }\end{array}$ \\
\hline Producto Interior Bruto 1997 & $\begin{array}{l}\text { World Development Indicators, Banco } \\
\text { Mundial. }\end{array}$ \\
\hline $\begin{array}{l}\text { Sector agrícola como tanto por ciento } \\
\text { del PIB 1997 }\end{array}$ & $\begin{array}{l}\text { World Development Indicators, Banco } \\
\text { Mundial. }\end{array}$ \\
\hline $\begin{array}{l}\text { Exportaciones de bienes y servicios como } \\
\text { tanto por ciento del PIB 1997 }\end{array}$ & $\begin{array}{l}\text { World Development Indicators, Banco } \\
\text { Mundial. }\end{array}$ \\
\hline
\end{tabular}

\section{Bibliografía}

Bardham, Pranab (2001). Democracy and Development: A Complex Relationship. Institute of International Studies Working Papers. Berkeley: University of California. Borx, Carles; STOKES, Susan (2003). «Endogenous Democratization». World Politics, núm. 55, p. 517-49.

BRYNJOLFSSON, E.; YANG, S. (1996). «Information technology and productivity: A review of the literature». Advanced Computer, núm. 43, p. 179-214.

BRYNJOlfsSON, Eric; HitT, Lorin; M. YANG, Shinkyu (2000). «Beyond Computation: Information Technology, Organization Transformation, and Business Performance». The Journal of Economic Perspectives, núm. 14, p. 23-48.

BRYNJOlfsSon, Eric; Smith, Michael D.; Hu, Ju (2003). «Consumer Surplus in the Digital Economy: Estimating the Value of Increased Product Variety at Online Booksellers». Management Science, núm. 49, p. 1580-1596.

BRYNJOLFSSON, Eric; HITT, Lorit M. (1995). «Information technology as a factor of production: The role of differences among firms». Journal of Economic Innovation and New Technologies, núm. 3, p. 183-199.

CASTELls, Manuel (1997). La era de la información. Tres volúmenes. Madrid: Alianza Editorial.

Cross, Bill (1998). "Teledemocracy: Canadian Political Parties Listening to their Constituents», en CYNTHIA, Alexander J. y LesLie Pal, V. (eds.). Digital Democracy: Policy and Politics in the Wired World. Don Mills, Ontario: Oxford University Press.

Cynthia, Alexander J.; Leslie Pal, V. (eds.) (1998). Digital Democracy: Policy and Politics in the Wired World. Don Mills, Ontario: Oxford University Press. 
EDWARDS, Sebastian (2001). Information Technology and Economic Growth in the Emerging Economies. Ponencia presentada en la Conferencia BBVA-CEPAL.

Etzioni, A. (1992). «Teledemocracy. The electronic Town Meeting», The Atlantic, núm. 270, p. 34-39.

FounTAIN, Jane E. (2001). Building the Virtual State. Information Technology and Institutional Change. Washington, D.C.: Brookings Institution Press.

Greenan, Nathalie; Lhorthy, Yannick; Mairesse, Jacques. 2001. The Puzzling Relations Between Computer and the Economy. Cambridge, Mass: MIT Press.

Hoff, Jens; Horrocks, Ivan; Tops, Peter. (eds.) (2000). Democratic Governance and New Technology. Londres: Routledge/ECPR Studies in European Political Science.

Inglemard, R.; Welzel, C. (1999). Analyzing Democratic Change and Stability: A Human Development Theory of Democracy. Veröffentlichung der Abteilung Institutionen und sozialer Wandel des Forschungs-schwerpunkts Sozialer Wandel, Institutionen und Vermittlungsprozesse des Wissen-schaftszentrums Berlin für Sozialforschung (FS III 99-202).

JORGENSON, Dale W.; STIROH, Kevin (1995). «Computers and Growth». Journal of Economics of Innovation and New Technology, núm. 3, p. 295-316.

Kaufmann, D.; Kraay, A.; Mastruzzi, M. (2003). "Governance Matters III: Governance Indicators for 1996-2002». World Bank Policy Research Working Paper 3106.

Krugman, Paul (1994). Vendiendo prosperidad. Barcelona: Ariel.

LIPSET, S. M. (1959). "Some social requisites of democracy: Economic development and political legitimacy». American Political Science Review, núm. 53, p. 69-105.

MARTIN, Hagen (1997). «Elektronische Demokratie: Eine Herausforderung für politische Bildung». Journal für politische Bildung, núm. 3, p. 26-30.

Nordhaus, William D. (2001). Productivity Growth and the New Economy. NBER Documento de Trabajo 8097.

NorRIS, Pippa (2001). "Theories of "Digital Democracy" and "Civil Society"», en NorRIS, Pippa. Digital Divide. Cambridge, Mass: Cambridge University Press.

Ogden, Michael R. (1994). «Politics in a Parallel Universe. Is there a future for Cyberdemocracy?». Futures, núm. 26, p. 713-729.

Oliner, Stephen D.; SiCHEL, Daniel E. (2000). «The Resurgence of Growth in the Late 1990's: Is Information Technology the Story». The Journal of Economic Perspectives, núm. 14, p. 3-22.

POSTER, Mark (1995). Cyberdemocray: internet and the public sphere. University of California, Irvine. Disponible en http:/www.hnet.uci.edu/mposter/writings/ democ.html.

PRATS, Joan O. (2002a). Information Technologies, Integrated Governance Structures and Common Pool Resources Sustainability: the Case of the Mula River Basin. Ponencia preparada per la Segunda Conferencia Internacional del International Institute of Administrative Sciences (IIAS). Nueva Delhi (India), del 5 al 9 de noviembre.

- (2002b). E-Preparación: metodología y análisis. Documento no publicado.

Prats, Joan O.; Del Álamo, Óscar (2001). «Electronic Democracy: Origens, Perspectives, and Future of a New Way of Making Politics». Artículo presentado en la conferencia Development in Public Participation and Innovations in Community Governments. Universidad Autónoma de Barcelona, del 7 al 9 de junio.

PRZEWORSKI, Adam; LimONGI, Fernando (1997). «Modernization: Theories and Facts». World Politics, núm. 49, p. 155-83. 
RoDrik, Dani (2001). Four Simple Principles For Democratic Governance Of Globalization. Friedrich Ebert Foundation Papers. Disponible en http://ksghome.harvard.edu/ - .drodrik.academic.ksg/shortpieces.html

Rodrik, D.; Subramanian, A.; TrebBI, F. (2002). Institutions Rule: The Primacy of Institutions over Geography and Integration in Economic Development, CID Working Paper No. 97. Center for International Development, Harvard University.

SALDICH, Anne R. (1979). Electronic democracy: television's impact on the american political process. Nueva York: Praeger.

Sen, Amartya (2000). Development as Freedom. Oxford University Press.

Welzel, CH.; Inglehart, R. (2001). Human Development and the Explosion of Democracy: Variations of Regime Change across 60 societies. Paper en Institutionen und Sozialer Wandel. Wissenschaftszentrum Berlin für Sozialforschung. Disponible en: http://www.wz-berlin.de/iw/papers/iii01-202.pdf 